\title{
How Alobatnic Writese Feature Articles in Blog?
}

\author{
Azka Felicia Rianzi ${ }^{1(*)}$ \\ ${ }^{1}$ Jupenizer Institute \\ Raffles Hills, Cluster Beauty, Blok X, No. 6, Jakarta Timur, 13720, Indonesia \\ ${ }^{(*)}$ ristiazz@gmail.com
}

\begin{abstract}
A feature article gives information of human interest. Feature articles are generally the stories in newspapers and magazines other than straight news stories, editorials, or advertising. In addition, because of their human interest, they attempt to involve the reader emotionally. We have chosen feature articles from Alobatnic's blog that will provide a resource for those who are new to explorations of feature article, key terminology, brief definitions, and links to literature for further explorations.
\end{abstract}

Keywords : alobatnic; feature articles; online publising; 


\section{REFERENCES}

Setiawan, Adib Rifqi. (2013, March 10). Kirana Tanpa Sirna: sepanjang menggelinjang memahat maslahat. Alobatnic.blogspot.com. URL: https://Alobatnic.blogspot.com/2013/03/turoichan.html

Setiawan, Adib Rifqi. (2013, July 15). Menjilati Yuli: tegap menghibur walau dalam tangis. Alobatnic.blogspot.com. URL: https://Alobatnic.blogspot.com/2013/07/yuli.html

Setiawan, Adib Rifqi. (2013, August 26). Bathari Ekonomi: tak langsir dirisak penyinyir. Alobatnic.blogspot.com. URL: https://Alobatnic.blogspot.com/2013/08/sri.html

Setiawan, Adib Rifqi. (2013, October 17). Pangeran Ternistakan: kontroversi serta ambisi tiada henti. Alobatnic.blogspot.com. URL: https://Alobatnic.blogspot.com/2013/10/prabowo.html

Setiawan, Adib Rifqi. (2013, November 06). Cendekiawan Picisan: melintang lintang intan terabaikan. Alobatnic.blogspot.com. https://Alobatnic.blogspot.com/2013/11/yohanes.html

Setiawan, Adib Rifqi. (2014, May 26). Risalah Kasidah: bayang bayang satu sisi restoe boemi. Alobatnic.blogspot.com.

URL: https://Alobatnic.blogspot.com/2014/05/dhani.html

Setiawan, Adib Rifqi. (2014, June 18). Glam Male: kirana bunga manusia biasa. Alobatnic.blogspot.com. URL: https://Alobatnic.blogspot.com/2014/06/andra.html

Setiawan, Adib Rifqi. (2014, July 17). Karena Lelaki Bukan Pengecut: driven by you tie your mother down. Alobatnic.blogspot.com. URL: https://Alobatnic.blogspot.com/2014/07/brian.html

Setiawan, Adib Rifqi. (2014, September 05). Popularitas adalah Tuhan: memberikan penghiburan, menumbuhkan pengharapan. Alobatnic.blogspot.com. URL: https://Alobatnic.blogspot.com/2014/09/farrokh.html

Setiawan, Adib Rifqi. (2015, May 11). Butcah Mbeling: the mad one's behavior in motion. Alobatnic.blogspot.com. URL: $\quad$ https://Alobatnic.blogspot.com/2015/05/butcahmbeling-richard-phillips-feynman.html

Setiawan, Adib Rifqi, \& Ma'munah, Malikatul. (2015, May 27). Nun: bahadur menghibur melantan pengharapan. Alobatnic.blogspot.com. URL: https://Alobatnic.blogspot.com/2015/05/emha.html

Setiawan, Adib Rifqi. (2015, June 02). Melantan Warisan Kaisar Telantar: telantar, telantar, telantar, terlantan. Alobatnic.blogspot.com. URL: https://Alobatnic.blogspot.com/2015/06/ibrahim.html

Setiawan, Adib Rifqi. (2016, January 22). Ionia: lingkungan pemula gelora kajian keilmuan. Alobatnic.blogspot.com. URL: https://Alobatnic.blogspot.com/2016/01/ionia.html 
Setiawan, Adib Rifqi. (2016, February 11). Mike Shinoda. Alobatnic.blogspot.com. URL: https://Alobatnic.blogspot.com/2016/02/mike-shinoda.html

Setiawan, Adib Rifqi. (2016, February 17). PARIS: nothing in this world turn you on. Alobatnic.blogspot.com. URL: https://Alobatnic.blogspot.com/2016/02/paris.html

Setiawan, Adib Rifqi. (2016, July 23). Kamu Dalam aku: memuja yang dicinta, membela yang dinista. Alobatnic.blogspot.com. URL: https://Alobatnic.blogspot.com/2016/07/bom.html

Setiawan, Adib Rifqi, \& Amatullah. (2016, October 01). Berkarya Sebagai Diaspora: Ulas Kilas Nadirsyah Hosen. Alobatnic.blogspot.com. URL: https://Alobatnic.blogspot.com/2016/10/berkarya-sebagai-diaspora.html

Setiawan, Adib Rifqi. (2016, November 17). K2: mengenang sahabat yang hebat. Alobatnic.blogspot.com. URL: https://Alobatnic.blogspot.com/2016/11/kaka.html

Setiawan, Adib Rifqi. (2017, February 15). Butcah Chuniez: Puzzle Persinggungan Perjalanan dengan Eny Rochmwati Octaviani. Alobatnic.blogspot.com. URL: https://Alobatnic.blogspot.com/2017/02/thata.html

Setiawan, Adib Rifqi. (2017, February 24). Penerapan Pendekatan Saintifik Untuk Melatihkan Literasi Saintifik Dalam Domain Kompetensi Pada Topik Gerak Lurus Di Sekolah Menengah Pertama. Undergraduate Thesis. Universitas Pendidikan Indonesia. URL: http://repository.upi.edu/29074/

Setiawan, Adib Rifqi. (2017, March 23). Guru yang Menyapih: tak melayang dipuji, tak tumbang dicaci. Alobatnic.blogspot.com. URL: https://Alobatnic.blogspot.com/2017/03/nong.html

Setiawan, Adib Rifqi. (2017, April 29). My Way Rhapsody!: Islam Aries-Blackjack, Is Islam?. Alobatnic.blogspot.com.

URL: https://Alobatnic.blogspot.com/2017/04/islam.html

Setiawan, Adib Rifqi. (2017, May 21). Itz Spring Voice: andai aku tak mengaguminya .... Alobatnic.blogspot.com. URL: https://Alobatnic.blogspot.com/2017/05/itz.html

Setiawan, Adib Rifqi. (2017, June 03). Meniti Ilmuwati: catatan peran perempuan dalam pembahasan alam. Alobatnic.blogspot.com. URL: https://Alobatnic.blogspot.com/2017/06/meniti-ilmuwati-scholaristi.html

Setiawan, Adib Rifqi. (2017, July 15). Hope, Faith, Love: A Brief Story of Yuli Rahmawati. Alobatnic.blogspot.com. URL: https://Alobatnic.blogspot.com/2017/07/hope-faithlove.html

Setiawan, Adib Rifqi. (2017, September 22). Mengonstruksi rancangan soal domain kompetensi literasi saintifik siswa smp kelas viii pada topik gerak lurusMengonstruksi rancangan soal domain kompetensi literasi saintifik siswa smp kelas viii pada topik gerak lurus. Wahana Pendidikan Fisika (WaPFi), 2(2), 44-8. URL: http://ejournal.upi.edu/index.php/WapFi/article/view/8277 
Setiawan, Adib Rifqi. (2018, January 01). Pantat Perekat Umat: Modal Erotic Zaskia Gotik. Alobatnic.blogspot.com. URL: https://Alobatnic.blogspot.com/2018/01/pantatperekat-umat.html

Setiawan, Adib Rifqi. (2018, January 05). This is Venice: tak lelah meniti tatanan, tak jengah menata titian. Alobatnic.blogspot.com. URL: https://Alobatnic.blogspot.com/2018/01/islam-aries-blackjack.html

Setiawan, Adib Rifqi. (2018, January 08). Islām Aries-Blackjack, is Islām?. Alobatnic.blogspot.com. URL: https://Alobatnic.blogspot.com/2018/01/islam-ariesblackjack.html

Setiawan, Adib Rifqi. (2018, January 11). Roman (Bukan) Picisan: tak lelah meniti tatatan, tak jengah menata titian. Alobatnic.blogspot.com. URL: https://Alobatnic.blogspot.com/2018/01/islam-aries-blackjack.html

Setiawan, Adib Rifqi. (2018, January 15). Clara $\mathrm{Ng}$ : an author for our time. Alobatnic.blogspot.com. URL: https://Alobatnic.blogspot.com/2018/01/islam-ariesblackjack.html

Setiawan, Adib Rifqi. (2018, January 16). Amazing Grace: Satu Sisi Religi Grace Natalie. Alobatnic.blogspot.com. URL: https://Alobatnic.blogspot.com/2018/01/amazinggrace.html

Setiawan, Adib Rifqi. (2018, January 17). Laila Fariha Zein (a.k.a. Uus atau Febi): penyuluh peluh, peluluh keluh, peluruh keruh. Alobatnic.blogspot.com. URL: https://Alobatnic.blogspot.com/2018/01/laila-fariha-zein.html

Setiawan, Adib Rifqi. (2018, January 18). Dari Fallen Menjadi Vallen: Fall in Love Vallen in Love in Valentine. Alobatnic.blogspot.com. URL: https://Alobatnic.blogspot.com/2018/01/via-vallen.html

Setiawan, Adib Rifqi. (2018, January 19). Aisha: rebel heart living for love. Alobatnic.blogspot.com. URL: https://Alobatnic.blogspot.com/2018/01/aisha.html

Setiawan, Adib Rifqi. (2018, January 26). Remy Sylado 23761: konsisten dengan seribu kemuliaan.

Alobatnic.blogspot.com.

URL: https://Alobatnic.blogspot.com/2018/01/remy-sylado-23761.html

Setiawan, Adib Rifqi. (2018, February 08). Sekilas Mengelilingi Luas Geometri: Mengingat René Descartes, Mengenang Muhammad al-Ghazālī. Alobatnic.blogspot.com. URL: https://Alobatnic.blogspot.com/2018/02/sekilas-mengelilingi-luas-geometri.html

Setiawan, Adib Rifqi. (2018, February 27). Maryam Musfiroh: An Educator for Our Time. Alobatnic.blogspot.com. URL: $\underline{\text { https://Alobatnic.blogspot.com/2018/02/maryam- }}$ musfiroh.html 
Setiawan, Adib Rifqi. (2018, March 01). Ki Oza Kioza: A Rain Shine Made in Indonesia. Alobatnic.blogspot.com. URL: https://Alobatnic.blogspot.com/2018/03/roza-lailatulfitria-oza-kioza.html

Setiawan, Adib Rifqi. (2018, March 05). Breast Capital: Konsep Baru Duo Serigala, Modal Erotis, dan Payudara Perempuan. Alobatnic.blogspot.com. URL: https://Alobatnic.blogspot.com/2018/03/breastcapital.html

Setiawan, Adib Rifqi. (2018, March 08). Apresiasi Semadyana pada YoonA: Ketika YoonA mampir ke Parc des Princes. Alobatnic.blogspot.com. URL: https://Alobatnic.blogspot.com/2018/03/apresiasi-semadyana-pada-yoona.html

Setiawan, Adib Rifqi. (2018, March 09). Venice Min: yakin diri dan rendah hati memanfaatkan kecantikan. Alobatnic.blogspot.com. URL: https://Alobatnic.blogspot.com/2018/03/venice-min.html

Setiawan, Adib Rifqi. (2018, March 24). Hard to Say Happy Birthday Park Bom. Alobatnic.blogspot.com. URL: https://Alobatnic.blogspot.com/2018/03/hard-to-sayhappy-birthday-park-bom.html

Setiawan, Adib Rifqi. (2018, March 30). Menelusuri Nama Maryam: anyam sanggam, anyam azam.

URL: https://Alobatnic.blogspot.com/2018/03/menelusuri-nama-maryam.html

Setiawan, Adib Rifqi. (2018, April 01). Ternyata Majalah SANTRI Masih Terbit!: catatan pribadi mengenai penerbitan Majalah SANTRI edisi (10) April 2018. Alobatnic.blogspot.com. URL: https://Alobatnic.blogspot.com/2018/04/ternyatamajalah-santri-masih-terbit.html

Setiawan, Adib Rifqi. (2018, April 02). Rosa Amalia Iqony: paduan yakin diri dan rendah hati. Alobatnic.blogspot.com. URL: https://Alobatnic.blogspot.com/2018/04/rosaamalia-iqony.html

Setiawan, Adib Rifqi. (2018, April 04). Busana: pemantas raga, pelaras jiwa. Alobatnic.blogspot.com. URL: https://Alobatnic.blogspot.com/2018/04/busana.html

Setiawan, Adib Rifqi. (2018, April 10). Eny Rochmawati Octaviani: memberikan hiburan, menyuntikkan harapan. Alobatnic.blogspot.com. URL: https://Alobatnic.blogspot.com/2018/04/eny-rochmawati-octaviani.html

Setiawan, Adib Rifqi. (2018, April 11). Risalah Riḥlah (Catatan Penjelajahan): melestarikan tradisi bermadzhab. Alobatnic.blogspot.com. URL: https://Alobatnic.blogspot.com/2018/04/risalah- rihlah.html

Setiawan, Adib Rifqi. (2018, May 05). Girls, Love, War: A Brief Story of Rahwayana. Alobatnic.blogspot.com.

URL: https://Alobatnic.blogspot.com/2018/05/rahwayana.html 
Setiawan, Adib Rifqi. (2018, May 11). Mathematics: to write nor not to write. Alobatnic.blogspot.com.

URL: https://Alobatnic.blogspot.com/2018/05/mathematics.html

Setiawan, Adib Rifqi. (2018, May 19). Máthēmatnic: catatan perjalanan. Alobatnic.blogspot.com.

URL: https://Alobatnic.blogspot.com/2018/05/mathematnic.html

Setiawan, Adib Rifqi. (2018, May 19). Máthēmatnic: catatan perjalanan. Alobatnic.blogspot.com. https://Alobatnic.blogspot.com/2018/05/mathematnic.html

URL:

Setiawan, Adib Rifqi. (2018, May 20). Manunggaling Meyda Sefira-Hujan Safir. Alobatnic.blogspot.com.

URL: https://Alobatnic.blogspot.com/2018/05/manunggaling-meyda-sefira-hujan-safir.html

Setiawan, Adib Rifqi. (2018, June 13). Perjuangan Angan Perempuan: Obrolan dengan Grace Natalie Louisa. Alobatnic.blogspot.com. URL: https://Alobatnic.blogspot.com/2018/06/perjuangan-angan-perempuan.html

Setiawan, Adib Rifqi. (2018, June 13). Perjuangan Angan Perempuan: Obrolan dengan Grace Natalie Louisa. Alobatnic.blogspot.com. URL: https://Alobatnic.blogspot.com/2018/06/perjuangan-angan-perempuan.html

Setiawan, Adib Rifqi. (2018, June 15).Come Back Bom! [컴 백봄!]. Alobatnic.blogspot.com. URL: https://Alobatnic.blogspot.com/2018/06/come-back-bom.html

Setiawan, Adib Rifqi. (2018, June 20).al-Munqidz min al-Dholāl karya Abū Ḥāmid Muhammad al-Ghozālī: pembebas dari kesalahan menuju kebenaran berlandaskan keilmuan. Alobatnic.blogspot.com. URL: https://Alobatnic.blogspot.com/2018/06/almunqidz-min-al-dholal-karya-abu-hamid-muhammad-al-ghazali.html

Setiawan, Adib Rifqi. (2018, June 22).Fakhrun: a brief friendship (5 November $11-2$ Agustus 2014). Alobatnic.blogspot.com. URL: https://Alobatnic.blogspot.com/2018/06/fakhrun.html

Setiawan, Adib Rifqi. (2018, June 27).Drama Korea. Alobatnic.blogspot.com. URL: https://Alobatnic.blogspot.com/2018/06/drama-korea.html

Setiawan, Adib Rifqi. (2018, June 28).Dewi Perssik: dari sensasi ke sensasi hingga daya menghadapi suami. Alobatnic.blogspot.com. URL: https://Alobatnic.blogspot.com/2018/06/dewi-perssik.html

Setiawan, Adib Rifqi. (2018, July 03). Crazy Rhapsody: deep in my heartCrazy Rhapsody: deep in my heart. Alobatnic.blogspot.com. URL: https://Alobatnic.blogspot.com/2018/07/crazy-rhapsody.html

Setiawan, Adib Rifqi. (2018, July 22). Paul DeHart Hurd: menjadi-jadi dari biologi. Alobatnic.blogspot.com. URL: https://Alobatnic.blogspot.com/2018/07/paul-deharthurd.html 
Setiawan, Adib Rifqi. (2018, August 04). Debut Mengajar Biologi: mengenali diri, melantan lingkungan. https://Alobatnic.blogspot.com/2018/08/debut-mengajar-biologi.html

Setiawan, Adib Rifqi. (2018, August 11). Biology : My Story. Alobatnic.blogspot.com. URL: https://Alobatnic.blogspot.com/2018/08/biology-my-story.html

Setiawan, Adib Rifqi. (2018, September 16). Lola Zieta Azelien: paduan yakin diri dan rendah hati memanfaatkan kecantikan. Alobatnic.blogspot.com. URL: https://Alobatnic.blogspot.com/2018/09/lola-zieta-azelien.html

Setiawan, Adib Rifqi. (2018, September 25). Peran Biologi dalam Mendukung Pelaksanaan Jihād. Alobatnic.blogspot.com. URL: https://Alobatnic.blogspot.com/2018/09/peranbiologi-dalam-mendukung-pelaksanaan-jihad.html

Setiawan, Adib Rifqi. (2018, September 25). Peran Biologi dalam Mendukung Pelaksanaan Jihād. Alobatnic.blogspot.com. URL: https://Alobatnic.blogspot.com/2018/09/peranbiologi-dalam-mendukung-pelaksanaan-jihad.html

Setiawan, Adib Rifqi. (2018, September 30). Karen Armstrong: there is a rainbow in Islām. Alobatnic.blogspot.com. URL: $\quad$ https://Alobatnic.blogspot.com/2018/09/karenarmstrong.html

Setiawan, Adib Rifqi. (2018, October 09). Bergerak Menuju Tak Tentu: risalah rihlah khidmah 'ilmiyyah. Alobatnic.blogspot.com. URL: https://Alobatnic.blogspot.com/2018/10/bergerak-menuju-tak-tentu.html

Setiawan, Adib Rifqi. (2018, October 18). Ketika Ibrōhīm Mengajak untuk Mengamati Alam: dari dorongan penasaran sampai gairah berserah. Alobatnic.blogspot.com. URL: https://Alobatnic.blogspot.com/2018/10/ketika-ibrohim-mengajak-untukmengamati.html

Setiawan, Adib Rifqi. (2018, October 25). Hukum Alam, Kehendak Bebas, Perilaku Manusia. Alobatnic.blogspot.com. URL: https://Alobatnic.blogspot.com/2018/10/hukum-alamkehendak-bebas-perilaku-manusia.html

Setiawan, Adib Rifqi. (2018, October 25). Into the New World (다시 만난 세계): proposal for the principle of least action in the simple life. Alobatnic.blogspot.com. URL: https://Alobatnic.blogspot.com/2018/10/into-the-new-world.html

Setiawan, Adib Rifqi. (2018, November 10). Pain Man or Fine Man?: Apa sih yang Istimewa dari Richard Phillips Feynman?. Alobatnic.blogspot.com. URL: https://Alobatnic.blogspot.com/2018/11/richard-phillips-feynman-pain-man-or-fine$\underline{\text { man.html }}$

Setiawan, Adib Rifqi. (2018, November 13). Surely You're Joking, Mr. Feynman!: Adventures of a Curious Character. Alobatnic.blogspot.com. URL: https://Alobatnic.blogspot.com/2018/11/surely-youre-joking-mr-feynman.html 
Setiawan, Adib Rifqi. (2018, November 14). Six Not-So-Amazing Video. Alobatnic.blogspot.com. URL: https://Alobatnic.blogspot.com/2018/11/six-not-soamazing-video.html

Setiawan, Adib Rifqi. (2018, November 16). Segitiga Sama Cinta: who is the queen of the sciences: mathematics or biology? Alobatnic.blogspot.com. URL: https://Alobatnic.blogspot.com/2018/11/segitiga-sama-cinta.html

Setiawan, Adib Rifqi. (2018, November 29). Risalah Riḥlah. Alobatnic.blogspot.com. URL: https://Alobatnic.blogspot.com/2018/11/risalah-rihlah.html

Setiawan, Adib Rifqi, \& Louisa, Grace Natalie. (2018, December 11). Keadilan Untuk Semua, Keadilan Untuk Perempuan Indonesia. Alobatnic.blogspot.com. URL: https://Alobatnic.blogspot.com/2018/12/keadilan-untuk-semua-keadilan-untuk.html

Setiawan, Adib Rifqi. (2018, December 12). A Brief Note of Science Education Development in Indonesia. Alobatnic.blogspot.com. URL: https://Alobatnic.blogspot.com/2018/12/a-brief-note-of-science-education.html

Setiawan, Adib Rifqi. (2018, December 17). Grace Natalie, PSI, Poligami. Alobatnic.blogspot.com. URL: https://Alobatnic.blogspot.com/2018/12/grace-nataliepsi-poligami.html

Setiawan, Adib Rifqi. (2018, December 21). Upaya Melatih Literasi Saintifik melalui Pembelajaran Ilmu Pengetahuan Alam (IPA). Alobatnic.blogspot.com. URL: https://Alobatnic.blogspot.com/2018/12/upaya-melatih-literasi-saintifik.html

Setiawan, Adib Rifqi. (2018, December 21). Irma Rahma Suwarma. Alobatnic.blogspot.com. URL: https://Alobatnic.blogspot.com/2018/12/irma-rahma-suwarma.html

Setiawan, Adib Rifqi. (2018, December 24). Madrasah Tasywiquth Thullab Salafiyyah (TBS) Kudus. https://Alobatnic.blogspot.com/2018/12/madrasah-tasywiquth-thullab-salafiyyah-tbskudus.html

Setiawan, Adib Rifqi. (2018, December 28). Garis Besar Haluan Matematika: towards a natural system of the mathematical world: proposal for the principle of least action in teaching mathematics. Alobatnic.blogspot.com. URL: https://Alobatnic.blogspot.com/2018/12/garis-besar-haluan-matematika.html

Setiawan, Adib Rifqi. (2018, December 31). Mabādī 'Asyroh Fisika. Alobatnic.blogspot.com. URL: https://Alobatnic.blogspot.com/2018/12/mabadi-asyroh-fisika.html

Setiawan, Adib Rifqi. (2019, January 05). We Broke Up, We Rising Up: From 2015 MAMA to 2NE1's breakup to BL $\Lambda$ DКРІИК's riseup. Alobatnic.blogspot.com. URL: https://Alobatnic.blogspot.com/2019/01/we-broke-up.html

Setiawan, Adib Rifqi, \& Louisa, Grace Natalie. (2019, January 11). Politik Akal Sehat, Politik Kaum Muda Alobatnic.blogspot.com. URL: https://Alobatnic.blogspot.com/2019/01/politik-akal-sehat-politik-kaum-muda.html 
Setiawan, Adib Rifqi. (2019, January 14). Basic Islamic Studies for Children: basic islamic studies for children from my traditional islamic perspective. Alobatnic.blogspot.com. URL: https://Alobatnic.blogspot.com/2019/01/basic-islamic-studies-for-children.html

Setiawan, Adib Rifqi, \& Louisa, Grace Natalie. (2019, February 11). Politik Akal Sehat, Politik Kaum Muda Alobatnic.blogspot.com. URL: https://Alobatnic.blogspot.com/2019/02/musuh-utama-persatuan-indonesia.html

Setiawan, Adib Rifqi. (2019, April 12). Q \& A. Grace Natalie Louisa. [interview]. Alobatnic.blogspot.com. URL: https://Alobatnic.blogspot.com/2019/04/grace-natalielouisa.html

Setiawan, Adib Rifqi. (2019, April 26). Curahan Hati untuk Teman-Teman Santri. Alobatnic.blogspot.com. URL: https://Alobatnic.blogspot.com/2019/04/curahan-hatiuntuk-teman-teman-santri.html

Setiawan, Adib Rifqi. (2019, April 26). Q \& A. Lisa Randall. [interview]. Alobatnic.blogspot.com. URL: https://Alobatnic.blogspot.com/2019/04/q-lisa-randallinterview.html

Setiawan, Adib Rifqi. (2019, April 26). An Interview with Venice Min. Alobatnic.blogspot.com. URL: https://Alobatnic.blogspot.com/2019/04/an-interviewwith-venice-min.html

Setiawan, Adib Rifqi. (2019, April 26). Panduan Penulisan Artikel. Alobatnic.blogspot.com. URL: https://Alobatnic.blogspot.com/2019/04/panduan-penulisan-artikel.html 